\title{
Medium-voltage distribution feeders in closed-loop arrangement - neutral point grounding
}

\author{
G. Štumberger ${ }^{1}$, K. Deželak ${ }^{1}$, M. Rošer ${ }^{2}$, R. Škof ${ }^{2}$ and T. Kastelic ${ }^{3}$ \\ ${ }^{1}$ University of Maribor \\ Faculty of Electrical Engineering and Computer Science \\ Smetanova 17, 2000 Maribor (Slovenia) \\ Phone/Fax number:+3862220 7075, e-mail: gorazd.stumberger@uni-mb.si, klemen.dezelak@uni-mb.si \\ ${ }^{2}$ Elektro Celje d.d. \\ Vrunčeva 2a, 3000 Celje (Slovenia) \\ Phone/Fax number:+386 3420 1000, e-mail: miran.roser@elektro-celje.si, robert.skof@elektro-celje.si \\ ${ }^{3}$ Elektro Primorska d.d. \\ Erjavčeva 22, 5000 Nova Gorica (Slovenia) \\ Phone/Fax number:+386 5339 6700, e-mail: tomaz.kastelic@elektro-primorska.si
}

\begin{abstract}
This paper deals with medium-voltage networks where the closed-loop arrangement of feeders is used to improve the reliability of power supply. The paper focuses on the neutral point grounding in the medium-voltage loops. They are established by closing feeders, supplied form different transformers, in closed-loop arrangement. It is shown in the case when the neutral points of transformers supplying the feeders are grounded, the fault current at the fault location can be doubled, without to influence the neutral point current, which could cause safety problems.
\end{abstract}

\section{Key words}

Medium-voltage distribution network, feeders in closedloop arrangement, neutral point grounding.

\section{Introduction}

The closed-loop arrangements of feeder can be used to improve reliability of power supply, voltage profiles and to reduce transmission losses in in medium voltage networks. The closed-loop arrangements of feeders can be classified as loops of the type I, type II and type III [1]. The type I loops are established by the closed-loop arrangement of two feeder supplied from the same transformer. The type II loop is established by the closed-loop arrangement of two feeders supplied from two different transformers located in the same substation. However, when the two transformers are located in two different substations, the two feeders in the closed loop arrangement form the type III loop. The papers [1] and [2] deal primarily with the loops of type I. They focus on the unbalance analysis and feasibility studies related to upgrading the feeders from the radial open-loop arrangement to the closed-loop arrangement.
Control of the loop distribution systems with distributed generation is discussed in [3]. The voltage angles and amplitudes at both end of the loop are used to control the active and reactive power in [4].

This paper focuses on the medium-voltage distribution networks where the loops of the types II and III are established primarily to improve the power supply reliability of extremely sensitive loads. Unfortunately, the closed-loop arrangement of feeders influences also the operational properties and performances of protection systems. Thus, the protection systems must be modified in order to work properly with feeders in the closed-loop arrangement.

This paper focuses on the neutral point grounding in the loops of type II and III, where different transformers supply both feeders in the closed-loop arrangement. In the case study, it is shown how different groundings of the neutral points of both transformers influence the neutral point and fault currents. It is shown that when the neutral points of both transformers supplying the loop are grounded, the ground fault current at the fault location is doubled, while the neutral point current is unchanged when the operation with feeders in the closed-loop arrangement is compared with the operation with feeder in the open-loop arrangement.

\section{Neutral point grounding}

The term neutral point grounding is widely used in North America while in the rest of the world as well as IEC normally use the term neutral earthing. The problems related with the neutral point grounding are, among the other works, treated also in [5-7]. Considering different types and different values of the impedance that 
characterizes the element that connects the neutral point with the ground, it is possible to distinct among five different types of the neutral point grounding:

1. Isolated neutral point where an intentional connection between the neutral point and the ground does not exist;

2. Resistance grounding where a resistor with generally high resistance value is placed between the neutral point and the ground;

3. Reactance grounding where a reactor with generally low reactance value is placed between the neutral point and the ground;

4. Compensated grounding where a reactor with changing reactance that is applied to compensate the system capacitance is placed between the neutral point and the ground;

5. Direct grounding where the neutral point is connected directly to the ground.

The selection of an appropriate neutral point grounding depends on: technical properties related mainly to the power system function, overvoltages and fault currents; operational properties like maintenance and required operation under the fault; safety; investment coats and operation costs; local practices. The neutral point grounding must fulfill two contradictory requirements. The first one is the reduction of overvoltages while the second one is the reduction of earth fault currents. An overvoltage normally appears due to the lightning, switching and resonance in the system, and appearance of a ground fault and its elimination. An excessive overvoltage can lead the dielectric breakdown that can end with short-circuit. On the other hand, too high ground fault current can endanger people through the increased potential of conductive parts. It can thermally overload cable shielding and it can cause damage due to the arc the fault location. Through the induction it influences electric circuits in its vicinity.

The type of neutral point grounding depends primarily on the properties of the electricity network where it is installed and on the established local practice. According to the practice established in Slovenian medium-voltage electricity distribution networks, in the case of existing transformers, the neutral points at the $20 \mathrm{kV}$ side of transformers $110 \mathrm{kV} / 20 \mathrm{kV}$ are mostly resistance grounded through $80 \Omega$ resistors. However, in the last years many systems with resistance grounding were updated to the compensated grounding.

This paper focuses on the systems with resistance grounding. Discussed are some of the problems related with the neutral point grounding that appear when the network operation changes from the operation with the open-loop arrangement of feeders to the operation with closed-loop arrangement of feeders.

\section{Discussed system}

The discussed system is located in the substation Krško, Slovenia. It is shown in Fig. 1. The two transformers $110 \mathrm{kV} / 20 \mathrm{kV}$, marked with TR1 and TR2, supply the busbars S1 and S2 and through them the feeders SR1 and SR2, which operate in the open-loop arrangement or in the closed-loop arrangement. The neutral points of both transformers can be grounded either individually, using two $80 \Omega$ resistors, or the neutral points of both transformers can be connected and grounded together, using only one $80 \Omega$ resistor. Fig. 2 shows the discussed system in the form of a block diagram used in dynamic model. The measurement points at the terminals of both transformers are marked with M3 and M4 while the measurements points at both feeders are marked with M31 and M41. The switch shown in Fig. 2 is used to change between the operation of feeders in the open-loop arrangement and in the closed-loop arrangement. The position where the ground fault is simulated is clearly marked in Fig. 2.

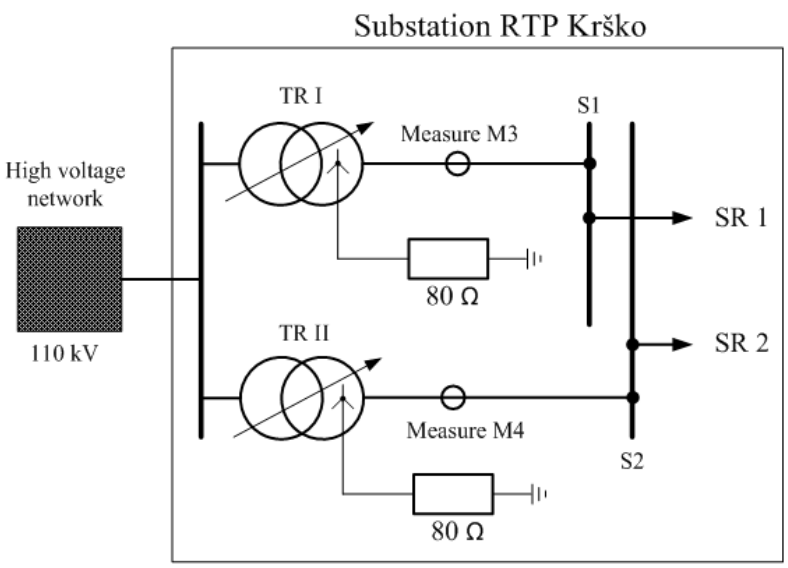

Fig. 1. Schematic presentation of discussed system

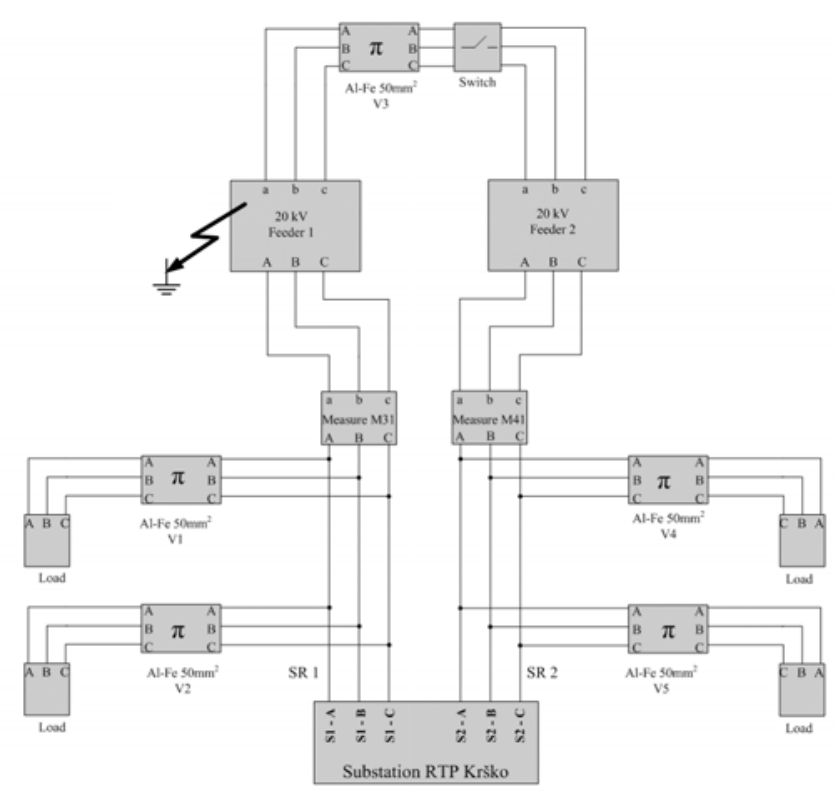

Fig. 2. Dynamic model of the discussed system

The dynamic model of the system, schematically shown in Figs. 1 and 2, is used to calculate the zero component current and the fault current at the fault location in the case when the neural points of the transformers are grounded individually and in the case when the neutral points are connected and grounded with only one resistor. 


\section{Results}

Figs. 3 to 6 show the time behaviors of the voltages $u$, the currents $i$, the active power $P$, and the reactive power $Q$ in the measurement points M3, M4, M31 and M41. They are given for the cases of operation where the neutral points of the two transformers are grounded individually and where the neutral points of transformers are connected together and grounded with only one resistor.
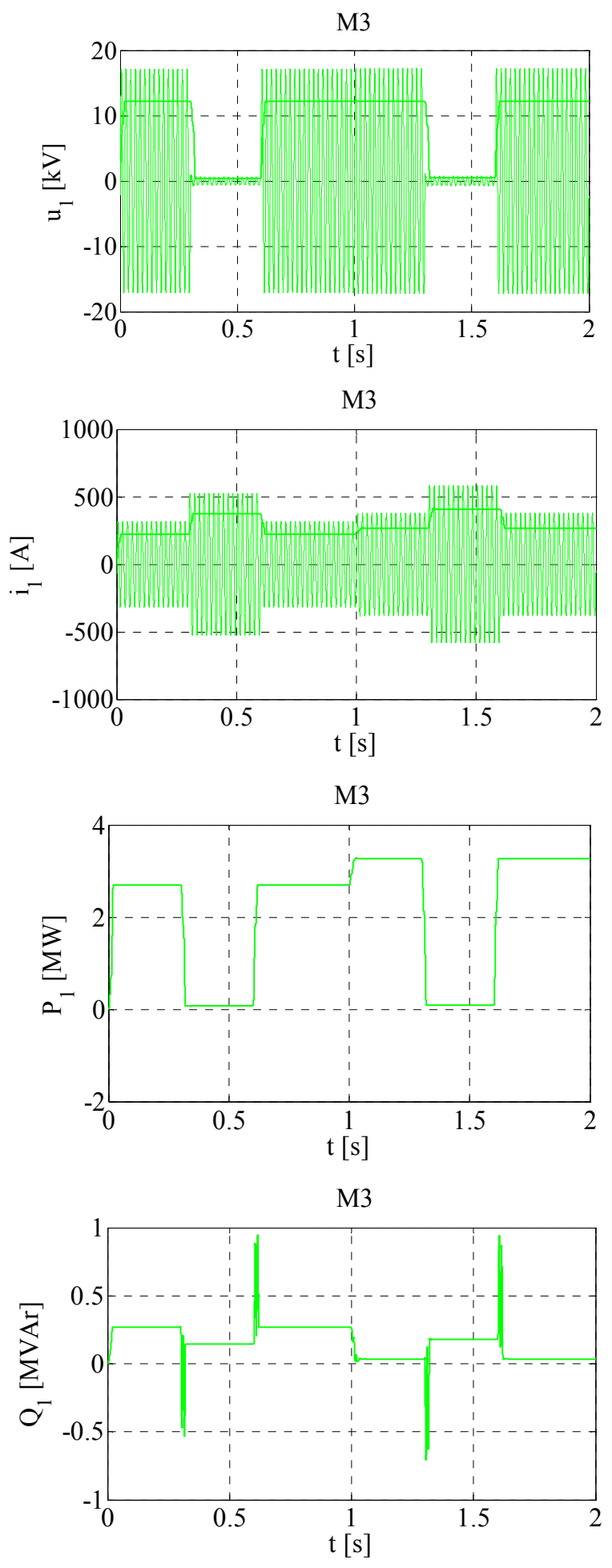

Fig. 3. Current $i_{1}$, voltage $u_{1}$, active power $P_{1}$ and reactive power $Q_{1}$ measured at measurement point M3
In the time interval between $0 \mathrm{~s}$ and $1 \mathrm{~s}$ the feeders are in the open-loop arrangement, while in the interval between $1 \mathrm{~s}$ and $2 \mathrm{~s}$ the feeders are in the closed-loop arrangement. The ground-fault appears between $0.3 \mathrm{~s}$ and $0.6 \mathrm{~s}$ for the open-loop arrangement of feeders and between $1.3 \mathrm{~s}$ and $1.6 \mathrm{~s}$ for the closed-loop arrangement of feeders.
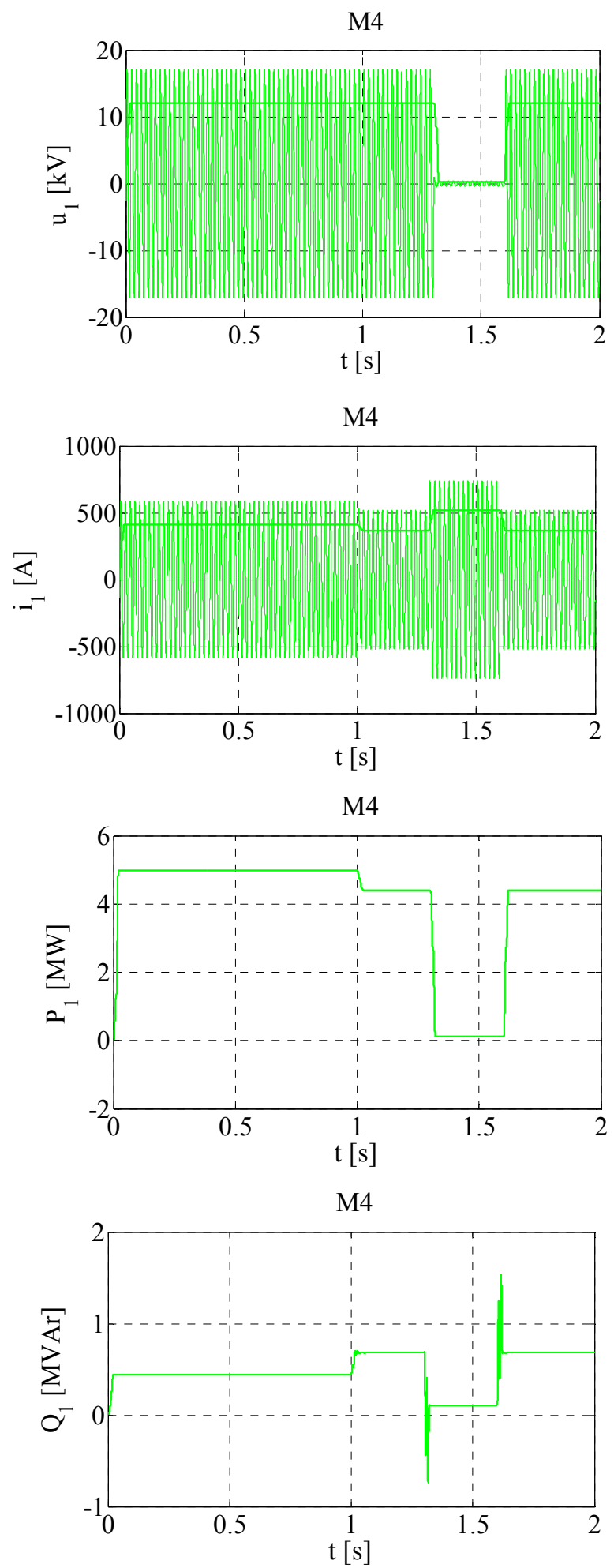

Fig. 4. Current $i_{1}$, voltage $u_{1}$, active power $P_{1}$ and reactive power $Q_{1}$ measured at measurement point M4 

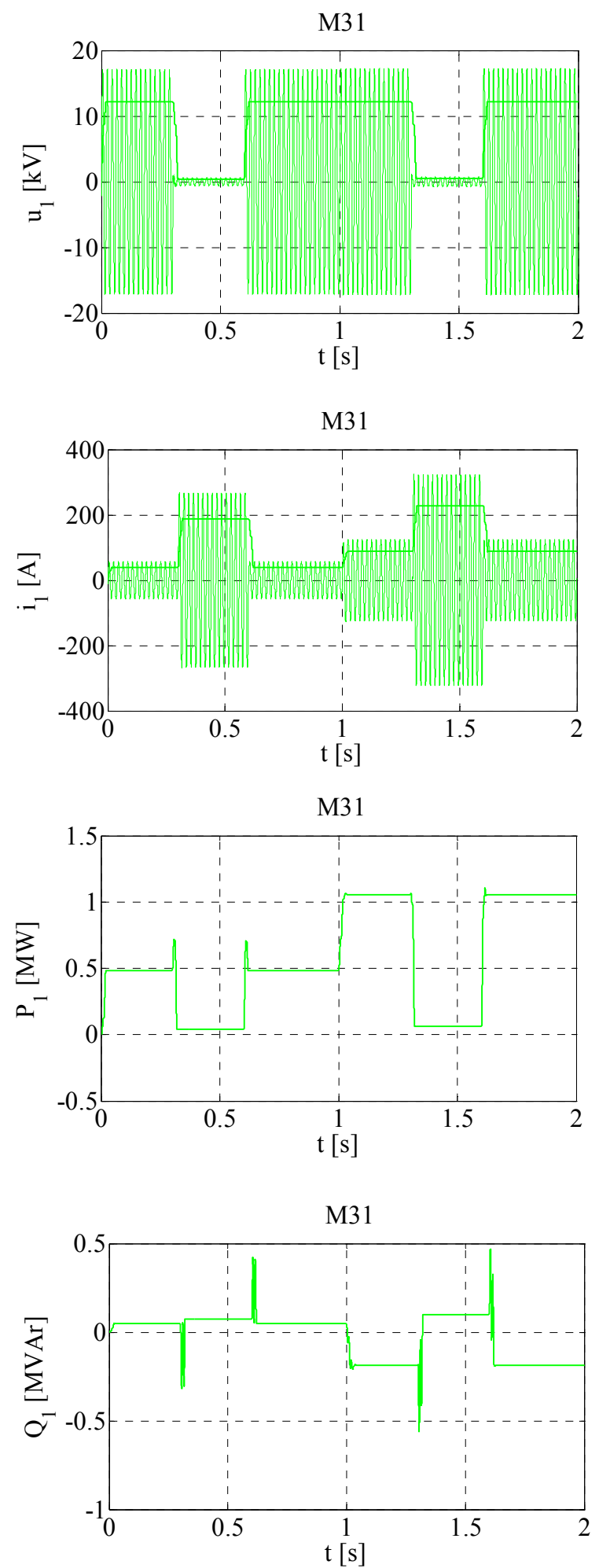

Fig. 5. Current $i_{1}$, voltage $u_{1}$, active power $P_{1}$ and reactive power $Q_{1}$ measured at measurement point M31

The transition from the open-loop arrangement of feeder to the closed-loop one, that appears as the time $1 \mathrm{~s}$, can be clearly seen in the currents $i_{1}$, the active power $P_{1}$ and the reactive power $Q_{1}$ shown in Figs. 3 to 6 . The changes in aforementioned variables are more expressed on individual feeders in measurement point M31 and M41 than on the busbars in measurements points M3 and M4. The values of $i_{1}$, and $P_{1}$ in M31 increase while the value of $Q_{1}$ decreases after the time $1 \mathrm{~s}$ (Fig. 5). The opposite situation can be seen in the measurement point M41, shown in Fig. 6.
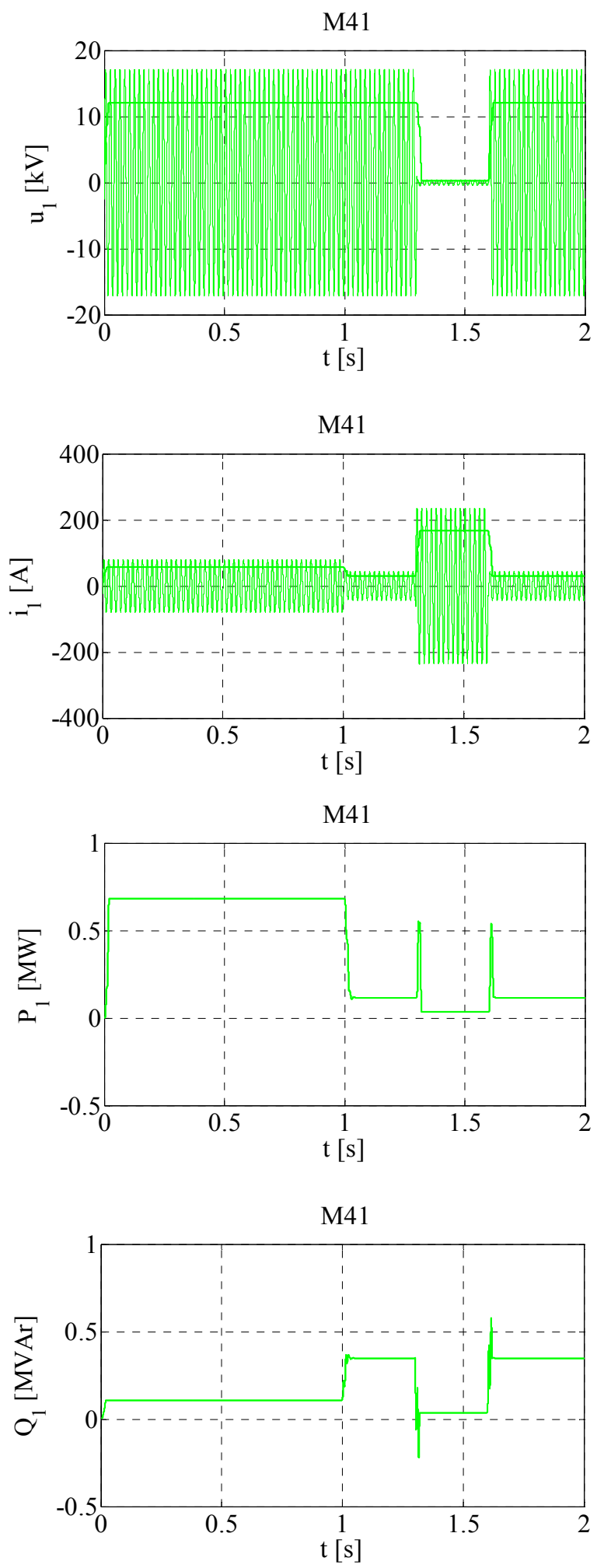

Fig. 6. Current $i_{1}$, voltage $u_{1}$, active power $P_{1}$ and reactive power $Q_{1}$ measured at measurement point M41

According to Fig. 1, the ground-fault appears in the feeder after the measurement point M31. Therefore, the ground fault that appears between $0.3 \mathrm{~s}$ and $0.6 \mathrm{~s}$, before the closed-loop arrangement of the feeders is established at the time $\mathrm{t}=1 \mathrm{~s}$, influences only $u_{1}, i 1, P_{1}$ and $Q_{1}$ in measurement points M3 and M31 shown in Figs. 3 and 5. The same variables in measurement points M4 and M41, shown in Figs. 4 and 6, are undisturbed due to the feeders 
in the open-loop arrangement. However, thus changes substantially after the time $1 \mathrm{~s}$, when the closed-loop arrangement of feeders is established. The ground-fault, that appears in the time interval between $1.3 \mathrm{~s}$ and $1.6 \mathrm{~s}$, influences $u_{1}, i 1, P_{1}$ and $Q_{1}$ in all measurement points (M3, M31, M4, M41) as shown in Figs. 3 to 6. However, the line currents, voltages, active power and reactive power, cannot give a deeper insight into condition in the neutral point and at the fault location. Therefore, the zero component current and the fault current must be included in the analysis.

The corresponding zero component currents $i_{0}$ as well as the fault currents $i_{\mathrm{f}}$ at the fault location are shown in Figs. 7 to 10 . They are given in the form of their time behaviors shown in Figs. 7 and 8 and with their rms (root mean square) values shown in Figs. 9 and 10.
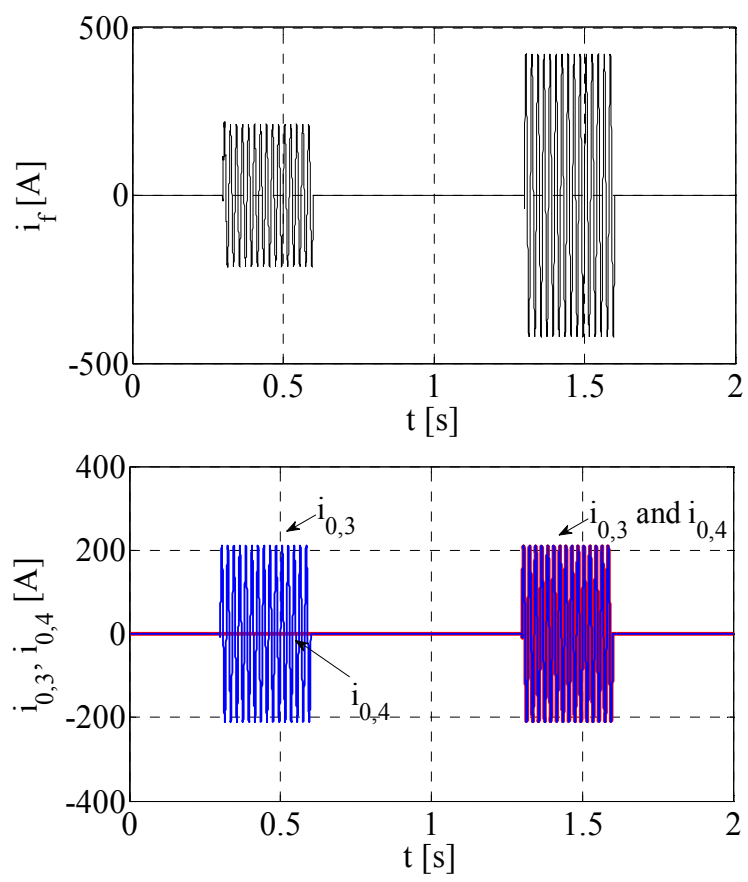

Fig. 7. Instantaneous values of the fault current $i_{\mathrm{f}}$ at the fault location and zero component currents at measurement points M3 and M4 $i_{0,3} i_{0,4}$ given for individually grounded neutral points of TR1 and TR2

In the time interval between $0 \mathrm{~s}$ and $1 \mathrm{~s}$ the feeders are in the open-loop arrangement, while in the interval between $1 \mathrm{~s}$ and $2 \mathrm{~s}$ the feeders are in the closed-loop arrangement. The ground-fault appears between $0.3 \mathrm{~s}$ and $0.6 \mathrm{~s}$ for the open-loop arrangement of feeders and between $1.3 \mathrm{~s}$ and $1.6 \mathrm{~s}$ for the closed-loop arrangement of feeders.

In the case of individually grounded neutral points of both transformers, the zero component current and the fault current appear during the ground-fault only in the faulted system 3 as shown in Figs. 7 and 9 for the time before $1 \mathrm{~s}$. The currents $i_{\mathrm{f}}$ and $i_{0,3}$ have similar time behaviors while $i_{0,4}=0$. At the time $1 \mathrm{~s}$ the closed-loop arrangement of feeders is established. After $1 \mathrm{~s}$, in the case of groundfault, the zero sequence currents $i_{0,3}$ and $i_{0,4}$ with similar time behavior appear in the neutral points of both transformers, while the fault current is almost doubled, as shown in Figs. 7 and 9. The doubled fault current causes easier ignition of a stronger arc that can lead to a fire. On the other hand the doubled fault current can substantially increase the potential of conductive parts along its path, which can endanger the health of people.
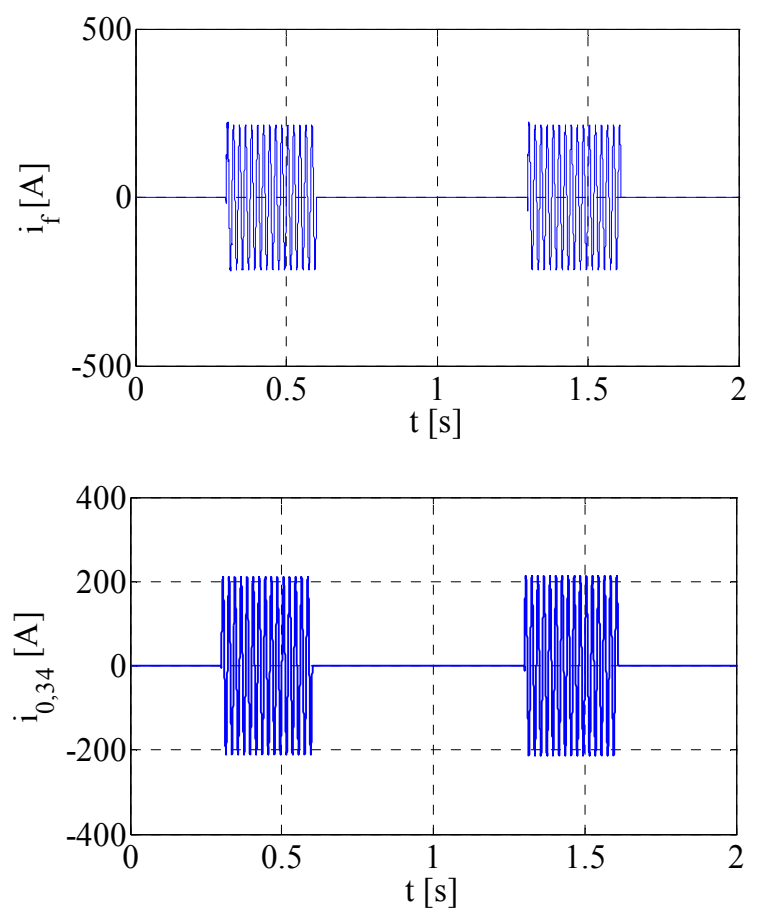

Fig. 8. Instantaneous values of the fault current $i_{\mathrm{f}}$ at the fault location and zero component currents at measurement points M3 and M4 $i_{0,3} i_{0,4}$ given for connected neutral points of TR1 and TR2 that are grounded with a single resistor
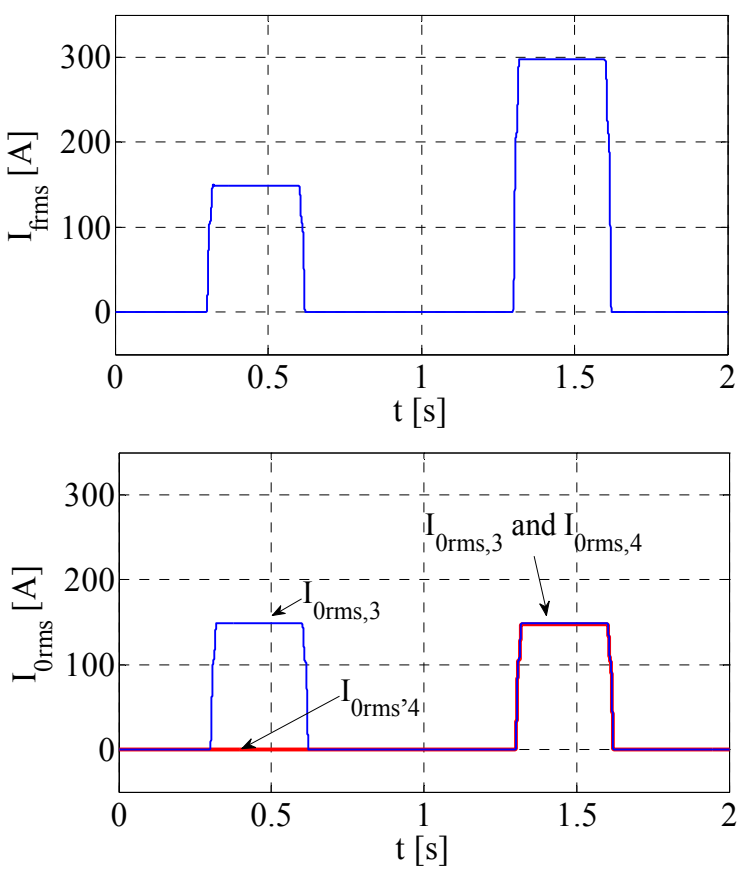

Fig. 9. Rms values of the fault current $I_{\text {frms }}$ at the fault location and zero component currents at measurement points M3 and M4 $I_{0 \mathrm{rms}, 3} I_{0 \mathrm{rms}, 4}$ given for individually grounded neutral points of TR1 and TR2

The results presented in Figs. 7 to 10 clearly show that, when the neutral points of both transformers are 
grounded individually and the feeders are in the closedloop arrangement, the earth-fault current at the fault location is doubled with respect to the feeders in the openloop arrangement. However, the currents through the neutral points are unchanged. This drawback can be eliminated in the case when the neutral points of both transformers are connected and grounded with only one resistor.
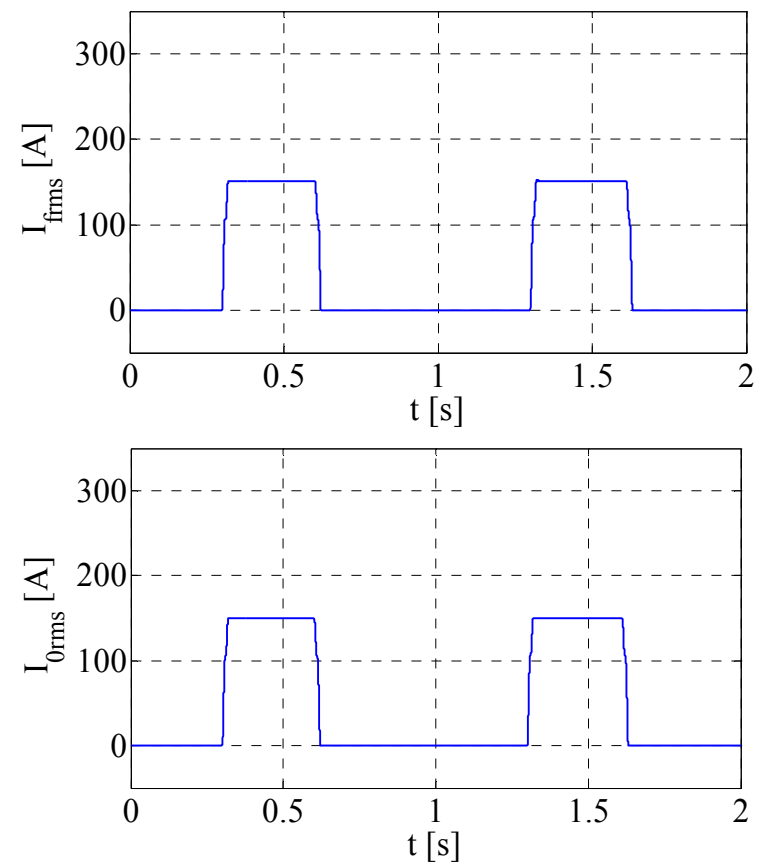

Fig. 10. Rms values of the fault current $I_{\text {frms }}$ at the fault location and zero component currents at measurement points M3 and M4 $I_{0 \mathrm{rms}, 3} I_{0 \mathrm{rms}, 4}$ given for connected neutral points of TR1 and TR2 that are grounded with a single resistor

\section{Conclusion}

Feeders in the closed-loop arrangement can substantially improve the power supply reliability, voltage profiles and electric power transmission losses. However, before closed-loop arrangement of feeder can be implemented, problems related with proper realization of protection should be solved.

\section{References}

[1] T. H. Chen, W. T. Huang, J. C. Gu, G. C. Pu, Y. H. Hsu, T. Y. Guo, Feasibility Study of Upgrading Primary Feeders from Radial and Open-Loop to Narmally Closed-Loop Arrangement, IEEE Transactions on Power Systems, vol. 19, no. 3, pp. $1308-1316,2004$.

[2] N. Okada, Verification of Control Method for a Loop Distribution System using Loop Power Flow Controller, in Proc. Power Systems Conference and Exposition (PSCE'06), pp. 2116 - 2123, 2006, Atlanta.

[3] W. C. Yang, W. T. Huang, J. Z. Chen, Unbalanced Analysis of Changing Medium-Voltage Distribution Fedders from Open-Loop to Closed-Loop Arrangement, in Proc. Third International Conference on Innovative Computing Information and Control, 2008, Dalian, China.

[4] G. Štumberger, K. Deželak, M. Rošer, R. Škof, T. Kastelic, Medium-voltage distribution feeders in open-loop and closed-loop arrangement, Proceedings of the International conference on renewable energies and power quality (ICREPQ' 12), 2012, Spain.

[5] C. Prévé, Protection of electrical networks, ISTE Ltd, 2006.

[6] P. Sorrells, A. Benitez, Power transformer neutral grounding reactor application and distribution feeder protection issues at CenterPoint Energy, 2005 58th Annual Conference for Protective Relay Engineers, 2005, pp. 27-39.

[7] Electrical network protection, Protection Guide, Schneider Electric Industries SAS, 2008. 